\title{
Utilização medicinal de espécies da família Cactaceae no semiárido do Brasil: Um estudo de caso na Paraíba
}

\section{Mariana Muniz Nunes ${ }^{1}$, Camilla Marques de Lucena ${ }^{2}$, Ezequiel da Costa Ferreira', Thamires Kelly Nunes

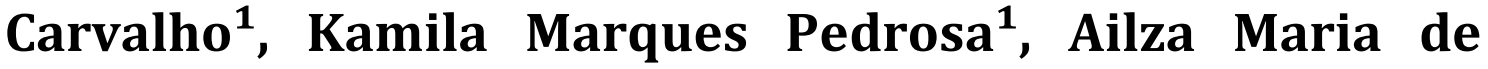 Lima-Nascimento', Guilherme Muniz Nunes ${ }^{1}$, Derly Pereira Brasileiro $^{1}$ e Reinaldo Farias Paiva de Lucena $^{1,2, *}$}

\author{
${ }^{1}$ Universidade Federal da Paraíba. Centro de Ciências Exatas e da Natureza. \\ Programa de Pós-Graduação em Desenvolvimento e Meio Ambiente. Campus I. João \\ Pessoa-PB, Brasil (CEP 58051-900). \\ ${ }^{2}$ Universidade Federal do Mato Grosso do Sul. Instituto de Biociências. Laboratório \\ de Etnobotânica. Campo Grande-MS, Brasil (CEP 79070-900). *E-mail: \\ reinaldolucena.dr@gmail.com.
}

Resumo. 0 presente estudo registrou o uso de espécies medicinais da família Cactaceae, com o objetivo de realizar um levantamento sobre a versatilidade, consenso de uso e importância das espécies, em seis comunidades rurais do semiárido do nordeste do Brasil. Os usos medicinais foram registrados a partir de entrevistas semiestruturadas com 182 chefes de família. A avaliação do conhecimento foi realizada através dos índices de Importância Relativa (IR) e Frequência Relativa de Citação (FRC). Para avaliação dos sistemas corporais com maior importância local foi utilizado o Fator de Consenso dos Informantes (FCI). Foram registradas seis espécies de Cactaceae de uso medicinal: Cereus jamacaru jamacaru (115 citações), Melocactus sp. (120), Opuntia ficus indica (5), Pilosocereus gounellei (19), Pilosocereus sp. (4) e Tacinga inamoena (4). 0 cálculo da Importância Relativa demonstrou que a espécie mais versátil foi $C$. jamacaru jamacaru (IR = 2). Com relação ao índice FRC a espécie Melocactus sp. obteve destaque (FRC =0,56). A partir do cálculo do FCI o Transtorno do sistema respiratório $(\mathrm{FCI}=0,96)$ obteve o maior valor. Portanto, foi possível observar que as comunidades rurais estudadas compartilham de um conhecimento relativamente síncrono em relação às espécies medicinais de Cactaceae pois, a maioria das citações foram para usos medicinais semelhantes, como, por exemplo, o tratamento de problemas do sistema respiratório. Deste modo, é possível notar a importância de estudos com espécies de uso medicinal da
Recebido

$26 / 12 / 2020$

Aceito

25/04/2021

Publicado

30/04/2021

Acesso aberto

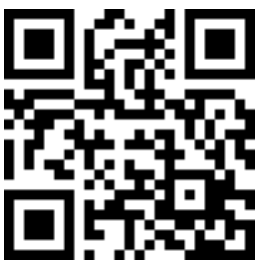

ORCID

(1) 0000-0002-9794-124X Mariana Muniz Nunes

(D) 0000-0002-5126-8969

Camilla Marques de

Lucena

ISSN 2359-1412/RBGAS-2020-0187/2021/8/18/39/593

Rev. Bras. Gest. Amb. Sustent.

http://revista.ecogestaobrasil.net 
Família Cactaceae, estudos estes que demonstram o poder terapêutico das plantas e valorizam o conhecimento popular das comunidades rurais do semiárido do nordeste do Brasil.

Palavras-chave: Cactos; Uso medicinal; Caatinga.

Abstract. Medicinal use of species of the Cactaceae family in the semiarid region of Brazil: A case study in Paraíba. The present study registered the use of medicinal species of the Cactaceae Family, with the objective of conducting a survey on the versatility, consensus of use and importance of the species, in six rural communities in the Semiarid Region of Northeast Brazil. Medicinal uses were recorded based on semi-structured interviews with 182 heads of households. Knowledge assessment was performed using the Relative Importance (RI) and Relative Quotation Frequency (RQF) indices. In order to assess body systems with greater local importance, the Informants Consensus Factor (ICF) was used. Six species of Cactaceae for medical use have been recorded: Cereus jamacaru jamacaru (115 citations), Melocactus sp. (120), Opuntia ficus indica (5), Pilosocereus gounellei (19), Pilosocereus sp. (4) and Tacinga inamoena (4). The calculation of Relative Importance demonstrated that the most versatile species was $C$. jamacaru jamacaru $(\mathrm{RI}=2)$. Regarding the RQF index, the species Melocactus sp. stood out $(\mathrm{RQF}=0.56)$. From the calculation of the RQF, the Respiratory System Disorder $(\mathrm{RQF}=0.96)$ obtained the highest value. Therefore, it was possible to observe that the rural communities studied share a relatively synchronous knowledge in relation to the medicinal species of Cactaceae because, most of the citations were for similar medicinal uses, such as, for example, the treatment of respiratory system problems. Thus, it is possible to note the importance of studies with species of medicinal use of the Cactaceae Family, studies that demonstrate the therapeutic power of plants and value the popular knowledge of rural communities in the semiarid region of northeastern Brazil.

Palavras-chave: Cacti; Medicinal use; Caatinga.

\author{
0000-0003-4758-7171 \\ Ezequiel da Costa \\ Ferreira \\ (1) 0000-0003-3847-1249 \\ Thamires Kelly Nunes \\ Carvalho \\ D) 0000-0003-1976-3545 \\ Kamila Marques \\ Pedrosa \\ D 0000-0002-8432-4533 \\ Ailza Maria de Lima- \\ Nascimento \\ (D) 0000-0003-2068-6436 \\ Guilherme Muniz \\ Nunes \\ (D) $0000-0002-1686-1257$ \\ Derly Pereira \\ Brasileiro \\ (D) 0000-0002-1195-4315 \\ Reinaldo Farias Paiva \\ de Lucena
}

\section{Introdução}

A utilização de plantas para fins medicinais nos sistemas médicos é resultado de um complexo processo de experimentações e validação no contexto dos povos tradicionais, ocorrendo quando as pessoas escolhem espécies vegetais naturalmente disponíveis em seus ambientes (Araújo et al., 2014). Esta validação é influenciada por fatores ambientais e socioeconômicos (Arruda et al., 2019; Kujawska et al., 2018; Quinlan e Quinlan, 2007). Em alguns locais, a aceitação do uso medicinal de plantas para cuidados primários já foi validada a ponto de ser recomendada por profissionais de saúde (AlonsoCastro et al., 2017). Pesquisas etnobotânicas que unem análises qualitativas e quantitativas são essenciais para auxiliar na seleção de espécies de plantas que necessitam ser valorizadas, conservadas e que possuem o potencial farmacológico (Linhares et al., 2014). Sendo assim, a validação do conhecimento popular e o investimento no isolamento e purificação de fito-constituintes e, principalmente, valorização e conscientização no uso e manejo de recursos naturais são cada vez mais imprescindíveis. 
Com base nisso, sistemas médicos mantidos por populações humanas têm sido alvo de investigações frequentes voltadas para entender como se dá a introdução de um repertório de plantas que são utilizadas e validadas pelas pessoas (Medeiros et al., 2017). Com a disponibilidade natural de espécies da família Cactaceae na Região Nordeste, como também de espécies introduzidas, as populações locais, ao longo do tempo, passaram a inserir algumas delas em seus sistemas médicos, assim como subexistir em função do uso de seus recursos úteis disponíveis e coletados localmente (Andrade et al., 2006). 0 aproveitamento geralmente acontece através do uso da planta inteira ou pela extração de determinadas partes.

Ademais, as comunidades locais que utilizam e manejam os recursos medicinais apresentam informações extremamente relevantes para auxiliar na seleção de espécies que são prioritárias para conservação e, além do conhecimento local, outro aspecto que deve ser ressaltado e considerado são as características socioeconômicas (ex. idade, sexo e profissão), pois as mesmas influenciam no conhecimento das pessoas e, consequentemente, devem ser considerados ao planejar as estratégias que auxiliam na conservação dos recursos naturais explorados (Silva et al. 2019).

Em estudos etnobotânicos com cactáceas no nordeste do Brasil que elencam fins medicinais, as espécies Cereus jamacaru DC. e Melocactus zehntneri (Britton \& Rose) Luetzelb normalmente são as que possuem maior concordância de uso tanto nos sistemas corpóreos, quanto nas partes das plantas que são utilizadas (Cartaxo et al., 2010; Lima-Nascimento et al., 2019). Para ambas já foram realizados estudos etnofarmacológicos atestando sua eficabilidade contra atividades microbianas infecciosas (Davet et al., 2009; Trentin et al., 2011).

Diante do exposto, o presente estudo teve como objetivo registrar e avaliar o conhecimento sobre o uso de espécies medicinais da família Cactaceae em seis comunidades rurais do semiárido nordestino, no estado da Paraíba. A avaliação do conhecimento foi realizada por meio dos índices de Importância Relativa (IR), Fator de Consenso dos Informantes (FCI) e Frequência Relativa de Citação (FRC).

\section{Material e métodos}

\section{Área de estudo}

0 estudo foi realizado em seis comunidades rurais, localizadas em cinco municípios no semiárido nordestino, no estado da Paraíba (Figura 1). A descrição das comunidades apresenta-se a seguir:

Comunidades São Francisco e de Tapera. Localizadas no município de Cabaceiras, na Mesorregião da Borborema e Microrregião do Cariri Oriental (07 29' 20" S e $36^{\circ} 17^{\prime} 14^{\prime \prime}$ ) ), a $400 \mathrm{~m}$ de altitude. Tal município abrange uma área de $452,920 \mathrm{~km}^{2} \mathrm{e}$ apresenta 5.035 habitantes (IBGE, 2010). Cabaceiras limita-se com os municípios de Campina Grande (norte), Barra de São Miguel (sul), Boqueirão (leste) e São João do Cariri (oeste). Dista 66,7 km do Município de Campina Grande (centro regional) e a $199 \mathrm{~km}$ de João Pessoa (capital do estado) (IBGE, 2010). Segundo a classificação de Köppen, o clima é do tipo Bsh - semiárido quente e, precipitação abaixo de $600 \mathrm{~mm}^{-a n o^{-1}}$ (IBGE, 2010). A vegetação é do tipo Caatinga hiperxerófila, tendo destaque para as Famílias Bromeliaceae e Cactaceae (Lucena et al. 2014).

As Comunidades de São Francisco e Tapera possuem atividade econômica baseada na criação de cabras e no cultivo de milho e feijão (Lucena et al. 2014). Na comunidade de Tapera, homens e mulheres, além de se dedicarem à agricultura, investem em atividades de turismo rural, o qual é realizado no Hotel Fazenda "Pai Mateus" (Pedrosa et al., 2020). Mensalmente os moradores das comunidades obtêm acesso aos cuidados médicos através de agentes de saúde. 
Comunidade Santa Rita. Localizada no município do Congo, na Microrregião do Cariri Ocidental e Mesorregião da Borborema, no Estado da Paraíba (07 47' 49" S e 36³9’36" 0), distando $212 \mathrm{~km}$ da capital, João Pessoa (IBGE, 2010). A população do Congo é de aproximadamente 4.687 habitantes distribuídos em uma área de 333,471 km², com municípios limítrofes de Serra Branca (ao norte), Coxixola e Caraúbas (ao leste), Camalaú e Sumé (ao oeste) e Santa Cruz, no Estado de Pernambuco (ao sul). Sua economia local é baseada na agricultura e pecuária (IBGE, 2010). O município encontra-se na Depressão Sertaneja, sendo uma parte de sua área (ao norte) inserida no Planalto da Borborema. 0 clima é tropical semiárido, com precipitação média anual de 431,8 $\mathrm{mm}$ e período chuvoso de novembro a abril. A vegetação é representada por Caatinga e alguns fragmentos de floresta caducifólia. Os tipos de solos encontrados são planossolos, bruno não cálcicos, podzólicos e litólicos.

A Comunidade Rural de Santa Rita apresenta economia baseada na produção e comercialização de alimentos como frutas, legumes e derivados de leite e carne. Com relação à saúde, os moradores da comunidade são acompanhados periodicamente por agente de saúde comunitário. Além disso, Santa Rita apresenta uma serra com cerca de $820 \mathrm{~m}$ de altitude, conhecida como Serra da Engabelada, é composta por geoformas de blocos de rochas graníticas (Sousa et al., 2012).

Comunidade Barroquinha e de Besouro. Situadas no Município de Lagoa, Mesorregião do Sertão e Microrregião de Catolé do Rocha, distante $394 \mathrm{~km}$ da capital. Sua população de 4.681 habitantes (2.377 na zona rural e 2.304 na zona urbana) encontra-se distribuída numa área de $177,901 \mathrm{~km}^{2}$ que tem como municípios limítrofes de Bom Sucesso, Jericó e Mato Grosso (ao norte), Pombal (ao sul), Paulista (ao leste) e Santa Cruz (ao oeste) (IBGE, 2010). Algumas comunidades rurais têm destaque no município, como Jatobá, Timbaúba, Jutubarana e Barroquinha, as quais são consideradas as maiores em termos de população residente.

Nas comunidades rurais de Barroquinha e Besouro quase cinquenta por cento dos moradores são agricultores e, além disso, outras ocupações como donas de casa, costureira, empregadas domésticas, funcionários públicos, motoristas, professores e vigilantes são registradas nas comunidades (Lucena et al.. 2012a).

Comunidade Várzea Alegre. Situa-se no Município de São Mamede, na Mesorregião do Sertão Paraibano e Microrregião do Seridó Ocidental, no centro do estado, a $6^{\circ} 55^{\prime} 37^{\prime \prime} \mathrm{S}$ de latitude e $37^{\circ} 05^{\prime} 45^{\prime \prime} \mathrm{O}$ de longitude. Possui 7.748 habitantes, sendo 3.837 homens e 3.911 mulheres, em uma área de $531 \mathrm{~km}^{2}$ (IBGE, 2010). 0 acesso pode ser feito a partir de João Pessoa pela Rodovia Federal BR-230 no sentido leste-oeste com um percurso de aproximadamente $278 \mathrm{~km}$. Limita-se com Ipueira (RN) e Várzea (ao norte), com Várzea e Santa Luzia (ao leste), Areia de Baraúnas, Passagem e Quixaba (ao sul) e Patos e São José de Espinharas (ao oeste), todas estas no Estado da Paraíba. O clima, segundo a classificação de Köppen é do tipo Bsh (semiárido quente) com chuvas de verão, temperatura média anual de $28^{\circ} \mathrm{C}$, e os solos são pedregosos.

A Comunidade Rural Várzea Alegre apresenta economia baseada na agricultura de sequeiro, ou seja, agricultura em período chuvoso, tendo destaque para o cultivo de feijão e milho (Lucena et al., 2012b). Na pecuária registra-se criação de gado, caprino e ovino. Mensalmente um agente de saúde visita cada unidade familiar e, além disso, os moradores podem ter acesso ao Posto de Saúde da Família (PSF), localizado em uma comunidade vizinha (Lucena et al., 2012b).

Comunidade Capivara. Localizada no Município de Solânea, Mesorregião do Agreste e Microrregião do Curimataú Oriental, semiárido da Paraíba, nordeste do Brasil. Próximo a Solânea estão os Municípios de Casserengue, Serraria, Dona Inês, Remígio, 
Arara, Barra de Santa Rosa, Borborema, Bananeiras e Cacimba de Dentro e, além disso, está por volta de $138 \mathrm{~km}$ de distância da capital do estado (João Pessoa) (Ribeiro et al. 2014). 0 município apresenta 26.689 habitantes, sendo 7.357 residentes da área rural (Carvalho et al., 2019). Segundo Prado (2003) a vegetação é representada por espécies da Caatinga. Solânea está situada em uma altitude de cerca de $626 \mathrm{~m}$ e apresenta temperatura anual de $25^{\circ} \mathrm{C}$ (Carvalho et al., 2019).

A comunidade rural Capivara está dividida em três áreas diferentes (Capivara I, II e III), sendo composta por 60 residências no total (Carvalho et al., 2019). Capivara apresenta associação de agricultores, os quais investem em cultivo de milho e feijão (agricultura de sequeiro) e na criação de ovelhas e cabras (Carvalho et al., 2019). Além disso, mensalmente os profissionais de saúde realizam visitas nas residências da comunidade (Ribeiro et al., 2014).
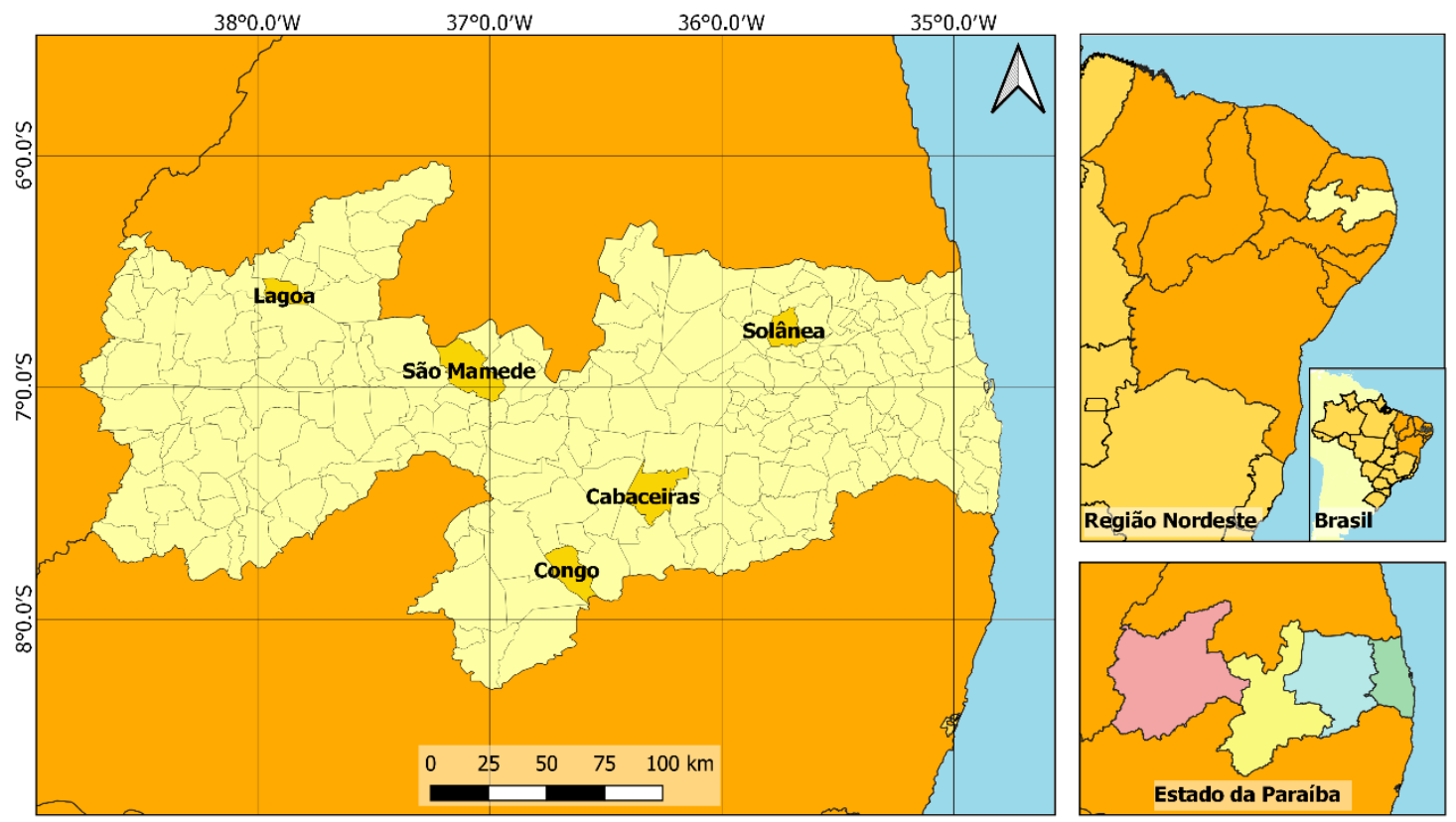

Figura 1. Mapa de localização dos municípios de Cabaceiras, Congo, Lagoa, São Mamede e Solânea, no estado da Paraíba, Nordeste do Brasil.

\section{Coleta de dados}

Todas as residências das comunidades estudadas foram visitadas e concomitantemente foi sendo explanado o objetivo do estudo. Aos mantenedores das famílias que aceitaram participar da pesquisa, foi solicitado a assinatura do Termo de Consentimento Livre e Esclarecido (TCLE) exigido pelo Conselho Nacional de Saúde por meio do Comitê de Ética em Pesquisa (Resolução no 196/1996). Ademais, o estudo foi aprovado pelo Comitê de Ética em Pesquisa com Seres Humanos (CEP) do Hospital Lauro Wanderley da Universidade Federal da Paraíba, o qual foi registrado pelo protocolo CEP/HULW no 297/2011.

A coleta de dados etnobotânicos foi realizada por meio de entrevistas semiestruturadas com o auxílio de formulário contendo perguntas sobre o uso, formas de uso, partes usadas e indicações terapêuticas das espécies de Cactaceae da região.

Foram entrevistados tanto homens como mulheres, em momentos distintos (Albuquerque et al., 2010), totalizando 182 participantes, sendo 58 em São Francisco (26 
homens e 38 mulheres), 36 em Santa Rita de Cima e Santa Rita de Baixo (12 homens e 24 mulheres), 23 em Barroquinha e Besouro (7 homens e 16 mulheres), 25 em Várzea Alegre (11 homens e 14 mulheres) e 40 em Capivara I, Capivara II, Capivara III (16 homens e 24 mulheres).

As espécies de Cactaceae citadas durante as entrevistas foram coletadas para serem herborizadas na forma de exsicata e, posteriormente, incorporadas no Herbário Jaime Côelho de Moraes (EAN) da Universidade Federal da Paraíba, no Centro de Ciências Agrárias (CCA).

\section{Análise de dados}

As indicações terapêuticas registradas nas entrevistas foram organizadas em 11 categorias estabelecidas pela Organização Mundial de Saúde (OMS, 2000), e utilizadas por Almeida e Albuquerque (2002), correspondendo a: Afecções não definidas ou dores não definidas; Doenças da pele e do tecido celular subcutâneo; Doenças das glândulas endócrinas, da nutrição e do metabolismo; Doenças do sistema osteomuscular e do tecido; Doenças infecciosas e parasitárias; Lesões externas e envenenamento; Neoplasia; Transtorno do sistema cardiovascular; Transtorno do sistema digestório; Transtorno do sistema geniturinário; e Transtorno do sistema respiratório.

Para verificar os sistemas corporais que demonstraram maior importância relativa local, foi calculado o Fator de Consenso dos Informantes (FCI) (Troter e Logan, 1986). Considerando que o valor máximo que uma subcategoria pode obter é 1 , quanto mais próximo ao valor máximo, maior será a semelhança entre os informantes sobre os usos medicinais das espécies de cactáceas citadas nas diferentes subcategorias:

$$
\mathrm{FCI}=(\text { nur }-\mathrm{nt}) /(\text { nur }-1)
$$

\footnotetext{
Onde:

$\mathrm{FCI}=$ Fator de Consenso entre os Informantes;

nur = número de citações no uso de registrado de cada subcategoria;

nt = número total de espécies incluídas nas subcategorias.
}

Foi calculado o índice de Importância Relativa (IR) para cada espécie de cactácea citada, com base em Bennett e Prance (2000). 0 cálculo foi realizado com base na seguinte fórmula: IR = NSC + NP, onde: IR = importância relativa; NSC: número de sistemas corporais registrados; $\mathrm{NP}=$ número de propriedades. $\mathrm{O}$ NSC é obtido pela razão NSCE/NSCEV, onde NSCE = número de sistemas corporais atribuídos a espécie considerada e NSCEV = número de sistemas corporais atribuídos a espécie mais versátil. 0 NP é obtido pela razão NPE/NPEV, onde NPE = número de propriedades atribuídas a espécie considerada e NPEV = número de propriedades atribuídas à espécie mais versátil. Tal método evidencia as espécies que se enquadram em um maior número de sistemas corporais, as que têm maior versatilidade ou maior número de indicações terapêuticas e, além disso, o valor máximo que uma espécie pode obter é 2 , sendo considerados altos valores de IR $\geq 1$.

A Frequência Relativa de Citação (FRC), com base em Tardío e Pardo-de-Santayana (2008) foi utilizada para identificar as espécies mais utilizadas e/ou conhecidas pelos informantes das comunidades. A fórmula é $\mathrm{FRC}=\mathrm{FC} / \mathrm{N}$, onde o $\mathrm{FC}$ é o número de informantes que mencionou o uso da espécie e $\mathrm{N}$ é o número total de informantes. Os resultados obtidos por este método variam de 0 (quando nenhum informante citou a espécie considerada) a 1 (quando todos os informantes citaram a espécie considerada). 
Para avaliar o grau de similaridade entre as espécies de cactáceas citadas pelos informantes nas comunidades foi realizado o teste de permutação ANOSIM-one way, com distância de Euclidean e permutação de 9999. Por meio deste teste é obtido um resultado $\mathrm{R}$ que varia de $-1 \mathrm{a}+1$, podendo indicar que não há diferença significativa entre os grupos $(R>0,25)$, que há alguma similaridade (em caso de valores entre $0,25<R<0,5$ ). Caso $R>$ 0,75 , indica valores distintos, com diferença total quando $\mathrm{R}=1$. Foi realizada uma análise de coordenadas principais (PCoA), utilizando a distância de Euclidean, para gerar um gráfico que representasse as diferenças de espécies citadas entre as comunidades. 0 programa utilizado para as análises foi o Past versão 3.22. Os dados foram tabulados no programa por meio de uma matriz de presença e ausência em código binário, onde 1 representava a menção de determinada espécie pelo informante e 0 representava a ausência da menção de determinada espécie. Os informantes foram considerados em agrupamentos conforme as comunidades.

\section{Resultados e discussão}

Foram registradas seis espécies de Cactaceae com uso medicinal, pertencentes a cinco gêneros. Cereus jamacaru DC. jamacaru (mandacaru), Melocactus sp. (coroa de frade), Opuntia ficus indica L. (Mill.) (palma), Pilosocereus gounellei (F.A.C. Weber) Byles \& Rowley gounellei (xique xique), Pilosocereus sp. (facheiro) e Tacinga inamoena (cumbeba; gogóia) (Tabela 1). Todas as espécies são nativas com exceção de $O$. ficus indica (Tabela 2).

Ao considerar outras famílias botânicas utilizadas em sistemas medicinais locais, estudos etnobotânicos e etnofarmacológicos demonstram que as espécies nativas têm maior relevância em relação às espécies exóticas no tratamento de diversas doenças (Medeiros et al., 2017).

Estudos etnobotânicos realizados no semiárido do Nordeste não citam a prática do cultivo de espécies nativas de Cactaceae nas comunidades rurais, contudo, a espécie exótica 0 . ficus indica tem sido cultivada por agricultores para ser utilizada como forragem e assim suprir necessidades coletivas em alimentar caprinos, bovinos e ovinos (Lucena et al. 2013, 2015).

Tabela 1. Indicações terapêuticas com seus respectivos sistemas corporais, parte usada, forma de uso e espécie de cactácea citada pelos informantes das comunidades estudadas no semiárido da paraíba, Nordeste do Brasil.

\begin{tabular}{|l|l|c|l|c|l|}
\hline Comunidade & $\begin{array}{l}\text { Indicações } \\
\text { terapêuticas }\end{array}$ & $\begin{array}{l}\text { Sistemas } \\
\text { corporais }\end{array}$ & Parte usada & $\begin{array}{l}\text { Forma } \\
\text { de uso }\end{array}$ & Espécie \\
\hline \multirow{5}{*}{ Barroquinha } & Colesterol & 6 & Polpa e raiz & A, B & Cereus jamacaru jamacaru \\
\cline { 2 - 6 } & $\begin{array}{l}\text { Doença do } \\
\text { coração }\end{array}$ & 6 & Raiz & $\mathrm{B}$ & Cereus jamacaru jamacaru \\
\cline { 2 - 6 } & Ferida no útero & 8 & Polpa & $\mathrm{A}$ & Cereus jamacaru jamacaru \\
\cline { 2 - 6 } & Gastrite & 7 & Polpa e raiz & $\mathrm{A}, \mathrm{E}$ & Cereus jamacaru jamacaru \\
\cline { 2 - 6 } & Gripe & 9 & Raiz & $\mathrm{B}$ & $\begin{array}{l}\text { Cereus jamacaru jamacaru e } \\
\text { Melocactus sp. }\end{array}$ \\
\cline { 2 - 6 } & Inflamação geral & 1 & Polpa & $\mathrm{A}$ & Cereus jamacaru jamacaru \\
\cline { 2 - 6 } & $\begin{array}{l}\text { Inflamação no } \\
\text { ovário }\end{array}$ & 1 & Polpa & $\mathrm{A}$ & Cereus jamacaru jamacaru \\
\cline { 2 - 6 } & $\begin{array}{l}\text { Inflamação nos } \\
\text { rins }\end{array}$ & 8 & Polpa & $\mathrm{A}$ & $\begin{array}{l}\text { Cereus jamacaru jamacaru } \mathrm{e} \\
\text { Melocactus sp. }\end{array}$ \\
\cline { 2 - 6 } & Tuberculose & 9 & Polpa & $\mathrm{E}$ & Melocactus sp. \\
\cline { 2 - 6 } & Úlcera & 7 & Polpa & $\mathrm{A}$ & Cereus jamacaru jamacaru \\
\cline { 2 - 6 } & Corrimento & 8 & Polpa & $\mathrm{D}$ & Cereus jamacaru jamacaru \\
\hline
\end{tabular}


Tabela 1. Continuação.

\begin{tabular}{|c|c|c|c|c|c|}
\hline Comunidade & $\begin{array}{l}\text { Indicações } \\
\text { terapêuticas }\end{array}$ & $\begin{array}{l}\text { Sistemas } \\
\text { corporais }\end{array}$ & Parte usada & $\begin{array}{l}\text { Forma } \\
\text { de uso }\end{array}$ & Espécie \\
\hline \multirow{12}{*}{ Besouro } & Gastrite & 7 & Polpa & $\mathrm{A}$ & Pilosocereus gounellei \\
\hline & Infecção & 1 & Raiz & $\mathrm{B}$ & Cereus jamacaru jamacaru \\
\hline & Inflamação & 1 & Polpa & A, D & Cereus jamacaru jamacaru \\
\hline & Cansaço & 4 & Polpa & $E$ & Cereus jamacaru jamacaru \\
\hline & Colesterol & 6 & Raiz & $\mathrm{B}$ & Cereus jamacaru jamacaru \\
\hline & Coqueluche & 5 & Polpa & $\mathrm{E}$ & Melocactus sp. \\
\hline & Dor na coluna & 4 & Raiz & $A, B$ & Cereus jamacaru jamacaru \\
\hline & Dor no corpo & 4 & Raiz & $\mathrm{A}, \mathrm{B}$ & Cereus jamacaru jamacaru \\
\hline & Gripe & 9 & Polpa e raiz & $\mathrm{B}, \mathrm{E}$ & $\begin{array}{l}\text { Cereus jamacaru jamacaru e } \\
\text { Melocactus sp. }\end{array}$ \\
\hline & Inchaço & 4 & Raiz & $\mathrm{B}$ & Cereus jamacaru jamacaru \\
\hline & Infecção & 5 & Raiz & $\mathrm{B}$ & Cereus jamacaru jamacaru \\
\hline & Infecção urinária & 8 & Raiz & $\mathrm{B}$ & Cereus jamacaru jamacaru \\
\hline \multirow{24}{*}{ Capivara } & Inflamação & 8 & $\begin{array}{l}\text { Entrecasca, } \\
\text { polpa e raiz }\end{array}$ & $\mathrm{A}, \mathrm{B}, \mathrm{F}$ & $\begin{array}{l}\text { Cereus jamacaru jamacaru e } \\
\text { Pilosocereus sp. }\end{array}$ \\
\hline & $\begin{array}{l}\text { Inflamação da } \\
\text { coluna }\end{array}$ & 4 & Raiz & $\mathrm{F}$ & Cereus jamacaru jamacaru \\
\hline & $\begin{array}{l}\text { Inflamação da } \\
\text { mulher }\end{array}$ & 8 & Raiz & $A, B$ & Cereus jamacaru jamacaru \\
\hline & $\begin{array}{l}\text { Inflamação nos } \\
\text { rins }\end{array}$ & 8 & Raiz & $\mathrm{A}, \mathrm{B}, \mathrm{C}$ & Cereus jamacaru jamacaru \\
\hline & $\begin{array}{l}\text { Mordida de } \\
\text { cobra }\end{array}$ & 10 & Raiz & $\bar{A}$ & Cereus jamacaru jamacaru \\
\hline & Pressão arterial & 6 & Fruto & $\bar{G}$ & Cereus jamacaru jamacaru \\
\hline & Próstata & 8 & Raiz & A, B & Cereus jamacaru jamacaru \\
\hline & Tosse & 9 & Polpa e raiz & E & $\begin{array}{l}\text { Cereus jamacaru jamacaru, } \\
\text { Melocactus sp., Pilosocereus } \\
\text { sp. e Tacinga inamoena }\end{array}$ \\
\hline & Úlcera & 7 & Polpa & $\mathrm{A}$ & Cereus jamacaru jamacaru \\
\hline & Verme & 5 & Fruto & $\mathrm{G}$ & Cereus jamacaru jamacaru \\
\hline & Alergia & 1 & Miolo & $\mathrm{A}$ & Cereus jamacaru jamacaru \\
\hline & Apêndice & 3 & Raiz & $\mathrm{A}, \mathrm{B}, \mathrm{C}$ & Cereus jamacaru jamacaru \\
\hline & Bronquite & 9 & Miolo & - & $\begin{array}{l}\text { Cereus jamacaru jamacaru e } \\
\text { Melocactus sp. }\end{array}$ \\
\hline & Câncer & 11 & Miolo & $\mathrm{H}$ & Melocactus sp. \\
\hline & Caroço & 2 & Baba e Miolo & $\bar{D}$ & $\begin{array}{l}\text { Cereus jamacaru jamacaru e } \\
\text { Melocactus sp. }\end{array}$ \\
\hline & Caroço no rosto & 2 & Galho & - & Cereus jamacaru jamacaru \\
\hline & Coluna & 4 & Miolo e raiz & $\mathrm{A}$ & Cereus jamacaru jamacaru \\
\hline & Diabete & 3 & Miolo & - & Cereus jamacaru jamacaru \\
\hline & $\begin{array}{l}\text { Doença da } \\
\text { mulher }\end{array}$ & 8 & Raiz & $\bar{B}$ & Cereus jamacaru jamacaru \\
\hline & $\begin{array}{l}\text { Doenças } \\
\text { venéreas }\end{array}$ & 5 & Miolo & - & Cereus jamacaru jamacaru \\
\hline & Fibromialgia & 4 & Miolo & $G$ & Cereus jamacaru jamacaru \\
\hline & Gripe & 9 & Fruto e Miolo & - & $\begin{array}{l}\text { Cereus jamacaru jamacaru e } \\
\text { Melocactus } \mathrm{sp} .\end{array}$ \\
\hline & $\begin{array}{l}\text { Regular ciclo } \\
\text { menstrual }\end{array}$ & 8 & Raiz & $\bar{A}$ & Cereus jamacaru jamacaru \\
\hline & Prisão de ventre & 7 & Fruto & $\mathrm{G}$ & Pilosocereus gounellei \\
\hline
\end{tabular}


Tabela 1. Continuação.

\begin{tabular}{|c|c|c|c|c|c|}
\hline Comunidade & $\begin{array}{l}\text { Indicações } \\
\text { terapêuticas }\end{array}$ & $\begin{array}{l}\text { Sistemas } \\
\text { corporais }\end{array}$ & Parte usada & $\begin{array}{l}\text { Forma } \\
\text { de uso }\end{array}$ & Espécie \\
\hline \multirow{18}{*}{ Santa Rita } & $\begin{array}{l}\text { Problema de } \\
\text { pele (eczema) }\end{array}$ & 2 & Miolo & - & Cereus jamacaru jamacaru \\
\hline & Próstata & 8 & Raiz & $\mathrm{B}$ & Cereus jamacaru jamacaru \\
\hline & Resfriado & 9 & Raiz & $\mathrm{B}, \mathrm{C}$ & Melocactus sp. \\
\hline & $\begin{array}{l}\text { Retirar espinhos } \\
\text { da pele }\end{array}$ & 10 & Baba e Miolo & $\mathrm{D}$ & $\begin{array}{l}\text { Melocactus sp., Opuntia ficus } \\
\text { indica, Pilosocereus gounellei, } \\
\text { Tacinga inamoena }\end{array}$ \\
\hline & Rins & 8 & Miolo e raiz & - & Cereus jamacaru jamacaru \\
\hline & Tosse & 9 & Fruto e Miolo & $E$ & $\begin{array}{l}\text { Cereus jamacaru jamacaru, } \\
\text { Melocactus sp. e Pilosocereus } \\
\text { sp. }\end{array}$ \\
\hline & Tubeculose & 9 & Miolo & - & Cereus jamacaru jamacaru \\
\hline & Tumor & 11 & Raiz & - & Melocactus sp. \\
\hline & Uretra & 8 & - & - & Tacinga inamoena \\
\hline & Verme & 5 & Miolo e raiz & $\mathrm{A}, \mathrm{H}$ & $\begin{array}{l}\text { Cereus jamacaru jamacaru e } \\
\text { Melocactus sp. }\end{array}$ \\
\hline & Vesícula & 3 & Raiz & $\mathrm{B}$ & Cereus jamacaru jamacaru \\
\hline & Anemia & 3 & Galhos & $\mathrm{G}$ & Pilosocereus sp. \\
\hline & Coqueluche & 5 & - & - & $\begin{array}{l}\text { Melocactus sp. e Opuntia ficus } \\
\text { indica }\end{array}$ \\
\hline & Dor na coluna & 4 & - & - & Cereus jamacaru jamacaru \\
\hline & Expectorante & 9 & Polpa & $\mathrm{E}$ & Melocactus sp. \\
\hline & Ferimento & 10 & - & - & Cereus jamacaru jamacaru \\
\hline & Furunco & 10 & - & - & Cereus jamacaru jamacaru \\
\hline & Infecção urinária & 8 & Raiz & $\mathrm{B}$ & Cereus jamacaru jamacaru \\
\hline \multirow{8}{*}{ São Francisco } & Inflamação & 1 & Raiz & $\bar{B}$ & Cereus jamacaru jamacaru \\
\hline & $\begin{array}{l}\text { Inflamação nos } \\
\text { rins }\end{array}$ & 8 & Raiz & $\bar{B}$ & Cereus jamacaru jamacaru \\
\hline & $\begin{array}{l}\text { Retirar os } \\
\text { espinhos da pele }\end{array}$ & 10 & Baba e polpa & $\overline{\mathrm{D}}$ & Pilosocereus gounellei \\
\hline & Reumatismo & 4 & Raiz & $\mathrm{B}$ & Cereus jamacaru jamacaru \\
\hline & Tosse & 9 & $\begin{array}{l}\text { Galhos e } \\
\text { polpa }\end{array}$ & $\bar{E}$ & $\begin{array}{l}\text { Cereus jamacaru sp., } \\
\text { Melocactus sp. e Opuntia ficus } \\
\text { indica }\end{array}$ \\
\hline & Uretra & 8 & Raiz & $A, B$ & Cereus jamacaru jamacaru \\
\hline & Verme & 5 & Polpa & $E$ & Melocactus sp. \\
\hline & Bexiga & 8 & Raiz & - & Cereus jamacaru jamacaru \\
\hline \multirow{5}{*}{ Tapera } & $\begin{array}{l}\text { Inflamação nos } \\
\text { rins }\end{array}$ & 8 & Raiz & $\mathrm{A}, \mathrm{B}, \mathrm{C}$ & $\begin{array}{l}\text { Cereus jamacaru jamacaru e } \\
\text { Pilosocereus gounellei }\end{array}$ \\
\hline & Uretra & 8 & Raiz & A, B & $\begin{array}{l}\text { Cereus jamacaru jamacaru e } \\
\text { Opuntia ficus indica }\end{array}$ \\
\hline & Bronquite & 9 & Polpa & $E$ & Melocactus sp. \\
\hline & Cansaço & 9 & Polpa & $\bar{E}$ & Melocactus sp. \\
\hline & Coqueluche & 5 & Polpa & $E$ & Melocactus sp. \\
\hline
\end{tabular}


Tabela 1. Continuação.

\begin{tabular}{|l|l|c|l|c|l|}
\hline Comunidade & $\begin{array}{l}\text { Indicações } \\
\text { terapêuticas }\end{array}$ & $\begin{array}{l}\text { Sistemas } \\
\text { corporais }\end{array}$ & Parte usada & $\begin{array}{l}\text { Forma } \\
\text { de uso }\end{array}$ & Espécie \\
\hline \multirow{5}{*}{ Várzea Alegre } & Dor de garganta & 9 & Fruto e polpa & E, G & Melocactus sp. \\
\cline { 2 - 6 } & Expectorante & 9 & Polpa & E & Melocactus sp. \\
\cline { 2 - 6 } & Gripe & 9 & Polpa & E & $\begin{array}{l}\text { Melocactus sp. e } \text { Opuntia ficus } \\
\text { indica }\end{array}$ \\
\cline { 2 - 6 } & $\begin{array}{l}\text { Inflamação } \\
\text { rins }\end{array}$ & 1 & Polpa e raiz & A, D & $\begin{array}{l}\text { Cereus jamacaru jamacaru e } \\
\text { Pilosocereus gounellei }\end{array}$ \\
\cline { 2 - 6 } & $\begin{array}{l}\text { Problema nos } \\
\text { pulmonar }\end{array}$ & 8 & Raiz & A & Cereus jamacaru jamacaru \\
\cline { 2 - 6 } & $\begin{array}{l}\text { Retirar os } \\
\text { espinhos da pele }\end{array}$ & 9 & Polpa & E & Melocactus sp. \\
\cline { 2 - 6 } & Sinusite & 9 & Baba & D & Pilosocereus gounellei \\
\cline { 2 - 6 } & Tosse & 9 & Polpa & E C & Cereus jamacaru jamacaru \\
\cline { 2 - 6 } & Verme & 5 & Polpa & E & Melocactus sp. \\
\hline
\end{tabular}

Forma de uso corresponde a: A: molho em água, B: decocção, C: infusão, D: uso tópico, E: lambedor; Sistemas corporais: 1 = Afecções não definidas ou dores não definidas, 2 = Doenças da pele e do tecido celular subcutâneo, 3 = Doenças das glândulas endócrinas, da nutrição e do metabolismo, $4=$ Doenças do sistema osteomuscular e do tecido conjuntivo, 5 = Doenças infecciosas e parasitárias, 6 = Transtorno do sistema cardiovascular, 7 = Transtorno do sistema digestório, 8 = Transtorno do sistema geniturinário, $9=$ Transtorno do sistema respiratório, $10=$ Lesões externas e envenenamento 11 = Neoplasia.

Foram registradas 267 citações de uso (166 citações a partir das mulheres e 101 dos homens), sendo organizadas em indicações terapêuticas (subcategorias medicinais) de acordo com o uso medicinal (Tabela 1).

As espécies mais citadas foram Melocactus sp. (coroa de frade) (120 citações), C. jamacaru jamacaru (mandacaru) (115 citações), P. gounellei (xique xique) (19 cit.), 0. ficus indica (palma) (5 cit.) e Pilosocereus sp. (facheiro) (4 cit.), T. inamoena (cumbeba; gogóia) (4 cit.) (Tabela 2).

Indicações terapêuticas como tosse (83 citações), gripe (21 citações), infecção urinária (20 cit.) e inflamação (17 cit.) foram as mais evidentes nas comunidades rurais (Tabela 1). Estudos etnobotânicos realizados com outras famílias botânicas também registram uma maior porcentagem de indicações de uso para o sistema respiratório (Gonçalves e Pasa, 2015).

A partir de uma revisão sistemática envolvendo dados de estudos com plantas medicinais que foram realizados no Brasil até 2011 foi possível registrar que para algumas indicações terapêuticas são utilizadas exclusivamente espécies nativas. 0 estudo também relata que a ausência de espécies exóticas no tratamento de doenças específicas (ex. sífilis que é tratada com a espécie nativa Cereus jamacaru DC.) auxilia na relevância e permanência das espécies nativas no sistema médico tradicional (Medeiros et al., 2017).

Transtorno do sistema respiratório foi o sistema corporal mais citado, seguido de transtorno do sistema geniturinário e lesões externas e envenenamento (Tabela 3).

Em todas as comunidades rurais, a polpa (miolo) com 159 citações foi a parte mais citada para o uso no preparo dos remédios caseiros, principalmente para o preparo de lambedor (93 citações), seguida da raiz (71 citações) sobretudo para o preparo de chá (44 citações), e baba (mucilagem) para uso tópico (12 cit.). Geralmente o uso medicinal de espécies de Cactaceae envolve a utilização de partes como raiz e polpa (miolo) (Lucena et al., 2012, 2013, 2014). 
Ao considerar a morfologia das espécies de Cactaceae (ex. arbóreo, arbustivo e subarbustivo), registradas no presente estudo, é notório que o uso em excesso das partes mais citadas (ex. polpa e raiz) pode causar um efeito prejudicial com relação conservação local dos indivíduos mais utilizados.

Com 120 citações Melocactus sp. foi a mais citada para o uso medicinal e, ao destacar que as espécies de tal gênero apresentam crescimento lento, não produz ramificação lateral e a propagação apenas por sementes (Correia et al., 2018), é indispensável uma atenção maior, visto que, são nativas e, algumas espécies correm risco de extinção (Silva et al., 2011; CNCFlora, 2012), como exemplo Melocactus glaucescens Buining \& Brederoo, que ocorre na Bahia e Melocactus lanssensianus P. J. Braun., que ocorrem na Paraíba e em Pernambuco (Batista et al. 2018; Zappi et al. 2018).

Contudo, ao considerar estudos realizados com espécies de outras famílias botânicas as partes mais citadas e/ou utilizadas pode ser raiz (Costa e Marinho, 2016), folhas (Ullah et al., 2020) e entrecasca (Linhares et al., 2014; Gonçalves e Pasa, 2015). Alguns trabalhos relatam que uma das possíveis explicações para o uso da folha, casca e/ou raiz serem proeminentes nos estudos etnobotânicos é a região e tipo de vegetação de ocorrência das espécies, pois em regiões semiáridas, como a caatinga, a maior parte da vegetação é do tipo caducifólia, e o uso da casca e da raiz é bem acentuado ao passo que, em ambientes como a Mata Atlântica (Zeni e Bosio, 2011) e Cerrado, a vegetação é do tipo perenifólia e, consequentemente, facilita o uso das folhas (Linhares et al., 2014). No entanto, há casos em que essa relação entre o tipo de ambiente e vegetação e a estrutura vegetal mais utilizada, podem não apresentar uma relação direta em função de outros fatores. Por exemplo, foi observado em áreas da Amazônia, que embora a vegetação seja perenifólia, ocorre um uso maior de estruturas como raiz e casca, neste caso, devido a altura do dossel que dificulta a coleta de folhas (Medeiros et al., 2013).

0 uso proeminente da polpa, com ênfase para a espécie Melocactus sp., no preparo dos remédios caseiros, pode suscitar um uso insustentável para o recurso, visto que impossibilita o desenvolvimento e a reprodução da planta.

A partir do cálculo da Importância Relativa foi possível observar que apenas uma espécie obteve alta versatilidade (isto é IR $\geq 1$ ) a partir das indicações dos informantes: $C$. jamacaru jamacaru (IR = 2) (Tabela 2). As espécies seguintes em maior versatilidade foram: Melocactus sp. (IR $=0,97)$ e P. gounellei (IR =0,62) (Tabela 2). Contudo, um trabalho realizado em uma comunidade do município do Parnaíba, estado do Piauí, o IR da espécie $C$. jamacaru jamacaru não foi tão relevante (0,39) (Brito et al., 2017) assim como no Distrito de Aratama, no Município de Assaré, Ceará (IR =0,33). Tais distinções em respeito a versatilidade desta espécie quando comparada ao observado no presente em estudo, provavelmente se dão pelo fato destes estudos terem se dado com uma maior diversidade de espécies em diferentes famílias botânicas, ocasionando um maior grupo de espécies para comparação.

As espécies com maior Frequência Relativa de Citação foram Melocactus sp. $(\mathrm{FRC}=0,56), C$. jamacaru jamacaru $(\mathrm{FRC}=0,46)$ e $P$. gounellei $(0,09)$. É possível, deste modo, observar uma inversão da primeira e da segunda espécie de maior destaque quando se compara a IR e a FRC, o que indica que, embora tenha havido um número menor de informantes citando o uso medicinal de C. jamacaru jamacaru em comparação a Melocactus sp., os informantes atribuíram uma maior diversidade de usos a $C$. jamacaru jamacaru. 
Tabela 2. Espécies de cactáceas medicinais nativas e exóticas registradas nas comunidades rurais de Barroquinha, Besouro, Capivara, São Francisco, Tapera e Várzea Alegre no semiárido da Paraíba, baseada no número de citações, na Importância Relativa (IR) e na Frequência Relativa de Citação (FRC).

\begin{tabular}{|c|c|c|c|c|c|c|c|}
\hline $\begin{array}{c}\text { Espécies/ } \\
\text { Voucher }\end{array}$ & $\begin{array}{c}\text { Nome } \\
\text { vernacular }\end{array}$ & $\begin{array}{c}\text { Hábito de } \\
\text { crescimento }\end{array}$ & Origem & $\begin{array}{c}\text { No de } \\
\text { citações }\end{array}$ & IR & FRC & Municípios \\
\hline $\begin{array}{c}\text { Cereus jamacaru } \\
\text { jamacaru (17619) }\end{array}$ & $\begin{array}{c}\text { Cardeiro; } \\
\text { mandacaru }\end{array}$ & Árvore & Nativa & 115 & 2 & 0,46 & $1,2,3,4,5$ \\
\hline $\begin{array}{c}\text { Melocactus sp. } \\
\text { (17572) }\end{array}$ & $\begin{array}{c}\text { Coroa-de- } \\
\text { frade }\end{array}$ & $\begin{array}{c}\text { Subarbusto } \\
\text { saxícola }\end{array}$ & Nativa & 120 & 0,97 & 0,56 & $1,2,3,4,5$ \\
\hline $\begin{array}{c}\text { Opuntia ficus } \\
\text { indica L. Miller } \\
(19769)\end{array}$ & Palma & Arbusto & Exótica & 5 & 0,52 & 0,02 & $1,2,4$ \\
\hline $\begin{array}{c}\text { Pilosocereus } \\
\text { gounellei (F.A.C. } \\
\text { Weber) Byles \& } \\
\text { Rowley (17629) }\end{array}$ & $\begin{array}{c}\text { Xlastrado; } \\
\text { Xique- }\end{array}$ & Arbusto & Nativa & 19 & 0,62 & 0,09 & $1,2,3,4$ \\
\hline $\begin{array}{c}\text { Pilosocereus sp. } \\
\text { (19616) }\end{array}$ & Facheiro & $\begin{array}{c}\text { Arvoreta/ } \\
\text { Arbusto }\end{array}$ & Nativa & 4 & 0,37 & 0,02 & $1,2,5$ \\
\hline $\begin{array}{c}\text { Tacinga inamoena } \\
\text { N.P. Taylor \& } \\
\text { Stuppy (19766) }\end{array}$ & Cumbeba & Subarbustiva & Nativa & 4 & 0,37 & 0,02 & 2,5 \\
\hline
\end{tabular}

Municípios: 1 = Cabaceiras, 2 = Congo, 3 = Lagoa, 4 = São Mamede, 5 = Solânea.

Os maiores valores do Fator de Consenso dos Informantes (FCI) foram associadas ao Transtorno do sistema respiratório $(\mathrm{FCI}=0,96)$, Transtorno do sistema digestório $(\mathrm{FCI}=0,92)$ e Transtorno do sistema geniturinário ( $\mathrm{FCI}=0,90)$ (Tabela 3). Assim como no presente estudo, o sistema corporal, Transtorno do sistema respiratório, também obteve destaque em outros estudos etnobotânicos realizados com espécies medicinais de diferentes famílias botânicas (Ribeiro et al., 2014; Brito et al., 2015). Além disso, foi possível registrar o segundo maior número de espécies de Cactaceae indicadas para Transtorno do sistema respiratório, o que demonstra um conhecimento síncrono em relação às espécies medicinais de Cactaceae que são eficazes para o tratamento que envolvem tal sistema corporal.

É comum observar na literatura um alto consenso para estes sistemas corporais e este consenso possivelmente se dá por esses sistemas envolverem enfermidades mais comuns, às quais as pessoas estão mais habituadas a lidar e tratar com o uso de plantas medicinais tornando, portanto, estes usos mais difundidos na comunidade.

A análise multivariada ANOSIM-one way demonstrou haver similaridade entre os conjuntos de cactáceas medicinais de todas as comunidades estudadas ( $R=0,06924$; $\mathrm{p}<0,0005)$. Por meio da análise de coordenadas principais (PCoA) é observada a similaridade entre as espécies de cactáceas medicinais citadas nas comunidades (Figura 2). 
Tabela 3. Fator de Consenso dos Informantes (FCI) das comunidades rurais Barroquinha, Besouro, Capivara, Santa Rita, São Francisco, Tapera e Várzea Alegre para o uso de espécies de cactáceas medicinais, na região do semiárido do estado da Paraíba, Nordeste do Brasil.

\begin{tabular}{|l|c|c|c|}
\hline Sistemas corporais & $\begin{array}{c}\text { Citações de uso em } \\
\text { cada sistema } \\
\text { corporal }\end{array}$ & $\begin{array}{c}\text { Espécies usadas } \\
\text { no sistema } \\
\text { corporal }\end{array}$ & FCI \\
\hline $\begin{array}{l}\text { Afecções não definidas ou dores não } \\
\text { definidas }\end{array}$ & 8 & 2 & 0,85 \\
\hline $\begin{array}{l}\text { Doenças da pele e do tecido celular } \\
\text { subcutâneo }\end{array}$ & 17 & 5 & 0,75 \\
\hline $\begin{array}{l}\text { Doenças das glândulas endócrinas, da } \\
\text { nutrição e do metabolismo }\end{array}$ & 4 & 2 & 0,66 \\
\hline $\begin{array}{l}\text { Doenças do sistema osteomuscular e do } \\
\text { tecido }\end{array}$ & 13 & 1 & 1 \\
\hline Doenças infecciosas e parasitárias & 16 & 3 & 0,86 \\
\hline Lesões externas e envenenamento & 19 & 1 & 1 \\
\hline Neoplasia & 2 & 1 & 1 \\
\hline Transtorno do sistema cardiovascular & 7 & 1 & 1 \\
\hline Transtorno do sistema digestório & 15 & 2 & 0,92 \\
\hline Transtorno do sistema geniturinário & 54 & 6 & 0,90 \\
\hline Transtorno do sistema respiratório & 123 & 5 & 0,96 \\
\hline
\end{tabular}

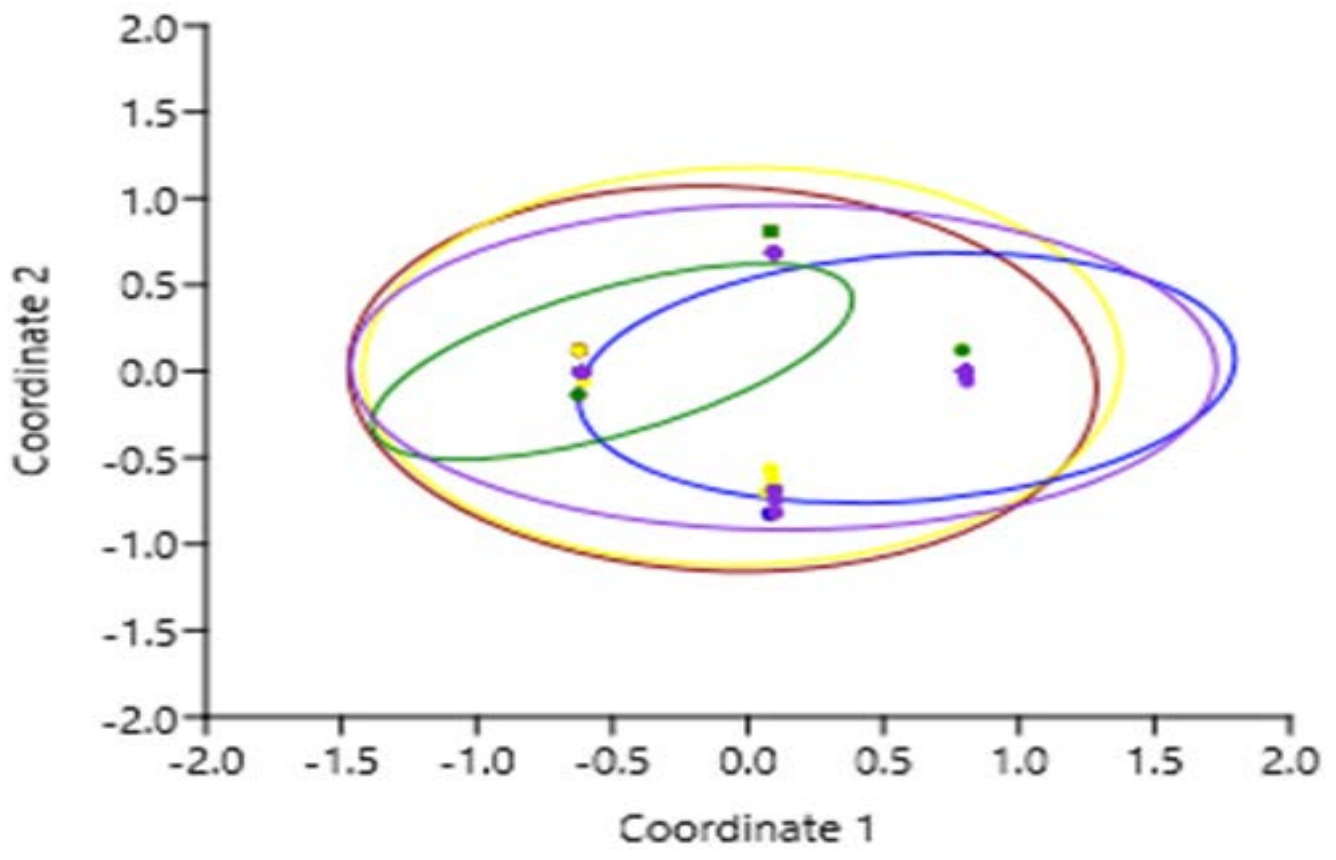

Figura 2. Análise de coordenadas principais (PCO) mostrando a similaridade entre as espécies citadas pelos informantes das comunidades rurais de cinco municípios do estado da Paraíba, Nordeste do Brasil. Vermelho = Cabaceiras; Amarelo = Congo; Azul = Lagoa; Verde = São Mamede; Roxo = Solânea . 


\section{Conclusão}

As comunidades estudadas apresentaram uso medicinal de cactáceas quase que exclusivamente com espécies nativas, com exceção apenas de 0 . ficus indica, que foi a única espécie exótica registrada, dado que aponta para a grande importância de espécies nativas em sistemas médicos locais. Por ser uma espécie cultivada localmente para uso forrageiro, o uso medicinal de $O$. ficus indica pode ser também um indício de como espécies exóticas introduzidas localmente para finalidades diversas, com o tempo, vão sendo também incorporadas às farmacopeias locais.

Os valores da Importância Relativa e da Frequência Relativa de Citação apresentaram destaque para C. jamacaru jamacaru e Melocactus sp. demonstrando que estas espécies apresentam um papel chave entre as cactáceas medicinais nas comunidades estudadas, sendo as espécies mais versáteis e mais conhecidas.

O número mais alto de citações, como também de valor do Fator de Consenso dos informantes para os Transtornos do sistema respiratório, pode ser um indício de que as enfermidades relacionadas a este sistema são mais comuns, conhecidas e tratadas pelas comunidades estudadas com o uso de plantas medicinais, bem como, que o conhecimento dos meios de tratamento deste grupo de doenças com uso de cactáceas é amplamente difundido entre a população.

As comunidades estudadas apresentam uma notável similaridade entre as espécies de cactáceas reconhecidas com propriedades medicinais, não tendo sido observado diferenças significativas nas espécies utilizadas entre as diferentes comunidades.

A extração frequente da polpa e das raízes das espécies de Cactaceae para fins medicinais é danosa a sobrevivência dos indivíduos. Portanto, partindo dos dados encontrados aqui, sugere-se que estudos futuros verifiquem a extração de partes destas plantas para uso medicinal, no sentido de verificar se este uso tem causado grande impacto aos indivíduos, e sugerir meios de um extrativismo sustentável destas espécies.

\section{Conflito de interesses}

Os autores declaram não haver conflito de interesses.

\section{Referências}

Almeida, C. F. C. B. R.; Albuquerque, U. P. Uso e conservação de plantas e animais medicinais no Estado de Pernambuco (Nordeste do Brasil): um estudo de caso. Interciencia, v. 27, n. 6, 276-285, 2002.

Araújo, T. A. S.; Melo, J. G.; Albuquerque, U. P. Plantas Medicinais. In: Albuquerque, U. P. (Ed.). Introdução à Etnobiologia. Recife: NUPPEA, 2014.

Arruda, H. L. S.; Santos, J. F. O.; Albuquerque, U. P.; Ramos, M. A. Influence of socioeconomic factors on the knowledge and consumption of firewood in the Atlantic Forest of Northeast Brazil. Economic Botany, v. 73, n. 1, p. 1-12, 2019. https://doi.org/10.1007/s12231-01909444-5

Alonso-Castro, A. J.; Domínguez, F.; Maldonado-Miranda, J. J.; Castillo-Pérez, L. J.; CarranzaÁlvarez, C.; Solano, E.; Isiordia-Espinoza, M. A.; Juárez-Vázquez, M. C.; Zapata-Morales, J. R.; Argueta-Fuertes, M. A.; Ruiz-Padilla, A. J.; Solorio-Alvarado, C. R.; Rangel-Velázquez, J. E.; Ortiz-Andrade, R.; González-Sánchez, I.; Cruz-Jiménez, G.; Orozco-Castellanos, L. M. Use of medicinal plants by health professionals in Mexico. Journal of Ethnopharmacology, v. 198, p. 81-86, 2017. https://doi.org/10.1016/j.jep.2016.12.038 
Andrade, C. T. S.; Marques, J. G. W.; Zappi, D. C. Utilização medicinal de cactáceas por sertanejos baianos. Revista Brasileira de Plantas Medicinais, v. 8, n. 3, p. 36-42, 2006.

Batista, F. R. C.; Almeida, E. M.; Alves, L. I. F.; Silva, P. K.; Neves, J. A. L.; Freitas, J. G. (Orgs.). Cactário Guimarães Duque: espécies da Coleção Botânica do INSA. Campina Grande: INSA, 2018.

Bennett, B. C.; Prance, G. T. Introduced plants in the indigenous pharmacopoeia of Northern South America. Economic Botany, v. 54, n. 1, p.90-102. 2000. https://doi.org/10.1007/BF02866603

Brito, M. F. M.; Lucena, R. F. P.; Cruz, D. D. Conhecimento etnobotânico local sobre as plantas medicinais: uma avaliação de índices quantitativos. Interciência, v. 40, n. 3, p. 156-164, 2015.

Cartaxo, S. L.; Almeida Souza, M. M.; Albuquerque, U. P. Medicinal plants with bioprospecting potential used in semi-arid Northeastern Brazil. Journal of Ethnopharmacology, $\quad$ v. $131, \quad$ n. 2 , $\quad$ p. 326-342, 2010. https://doi.org/10.1016/ j.jep.2010.07.003

Carvalho, T. K. N.; Lucena, C. M.; Lima, J. R. F.; Cruz, D. D.; Lucena, R. F. P. Local botanical knowledge of cacti in the Semi-Arid Region of Paraíba, Northeastern Brazil. Ethnobotany Research and Applications, v. 18, p. 1-11, 2019.

CNCFlora. Melocactus azureus in Lista Vermelha da flora brasileira versão 2012.2. Centro Nacional de Conservação da Flora. Disponível em: <http://cncflora.jbrj.gov.br/portal/ptbr/profile/Melocactus azureus>. Acesso em: 27 fev. 2021.

Correia, D.; Nascimento, E. H. S.; Santiago, L. G.; Gomes Filho, A. A. H.; Morais, J. P. S. Germinação in vitro de sementes de coroa-de-frade (Melocactus sp.). Brasília: EMBRAPA, 2018. (Boletim de Pesquisa e Desenvolvimento, 172).

Costa, J. C; Marinho, M. G. V. Etnobotânica de plantas medicinais em duas comunidades do município de Picuí, Paraíba, Brasil. Revista Brasileira de Plantas Medicinais, v. 18, n. 1, p. 125-134, 2016. https://doi.org/10.1590/1983-084X/15_071

Davet, A.; Virtuoso, S.; Dias, J. F. G.; Miguel, M. D.; Oliveira, A. B.; Miguel, O. G. Atividade antibacteriana de Cereus jamacaru DC, Cactaceae. Brazilian Journal of Pharmacognosy, v. 19, n. 2b, p. 561-564, 2009. https://doi.org/10.1590/S0102-695X2009000400009

Gonçalves, K. G.; Pasa, M. C. A etnobotânica e as plantas medicinais na Comunidade Sucuri, Cuiabá, MT, Brasil. Interações, v. 16, n. 2, p. 245-256, 2015. https://doi.org/10.1590/ 1518-70122015201

IBGE - Instituto Brasileiro de Geografia e Estatística. 2010. Disponível em: $<$ http://cidades.ibge.gov.br>. Acesso em: 15 out. 2020.

Kujawska, M.; Zamudio, F.; Montti, L.; Carrillo, V. P. Effects of landscape structure on medicinal plant richness in home gardens: Evidence for the environmental scarcity compensation hypothesis. Economic Botany, v. 72, n. 2, p.150-165, 2018. https://doi.org/10.1007/s12231-018-9417-3

Lima-Nascimento, A. M.; Bento-Silva, J. S.; Lucena, C. M.; Lucena, R. F. P. Ethnobotany of native cacti in the Northeast Region of Brazil: Can traditional use influence availability? Acta Botanica Brasilica, v. 33, n. 2, 2019. https://doi.org/10.1590/0102$33062019 a b b 0166$ 
Linhares, J. F. P.; Hortegal, E. V.; Rodrigues, M. I. A.; Silva, P. S. S. Etnobotânica das principais plantas medicinais comercializadas em feiras e mercados de São Luís, Estado do Maranhão, Brasil. Revista Pan-Amazônica de Saúde, v. 5, n. 3, p. 39-46, 2014. https://doi.org/10.5123/S2176-62232014000300005

Lucena, C. M.; Costa, G. M.; Sousa, R. F.; Carvalho, T. K. N.; Marreiros, N. A.; Alves, C. A. B.; Pereira, D. D.; Lucena, R. F. P. Conhecimento local sobre cactáceas em comunidades rurais na Mesorregião do Sertão da Paraíba (Nordeste do Brasil). Biotemas, v. 25, n. 3, p. 281-291, 2012a. https://doi.org/10.5007/2175-7925.2012v25n3p281

Lucena, C. M.; Costa, G. G. S.; Carvalho, T. K. N.; Guerra, N. M.; Quirino, Z. G. M.; Lucena, R. F. P. Uso e conhecimento de cactáceas no Município de São Mamede (Paraíba, Nordeste do Brasil). Revista de Biologia e Farmácia, v. esp., p. 121-134, 2012b.

Lucena, C. M.; Lucena, R. F. P; Costa, G. M.; Carvalho, T. K. N.; Costa, G. G. S.; Alves, R. R. N.; Pereira, D. D.; Ribeiro, J. E. S.; Alves, C. A. B.; Quirino, Z. G. M.; Nunes, E. N. Use and knowledge of Cactaceae in Northeastern Brazil. Journal of Ethnobiology and Ethnomedicine, v. 62, n. 9, p. 1-11, 2013. https://doi.org/10.1186/1746-4269-9-62

Lucena, C. M.; Carvalho, T. K. N.; Ribeiro, J. E. S.; Quirino, Z. G. M.; Casas, A.; Lucena, R. F. P. Conhecimento botânico tradicional sobre cactáceas no semiárido do Brasil. Gaia Scientia, v. 9, n. 2, p. 77-90, 2015.

Medeiros, P. M.; Haydée Ladio, A.; Albuquerque, U. P. Patterns of medicinal plant use by inhabitants of Brazilian urban and rural areas: A macroscale investigation based on available literature. Journal of Ethnopharmacology, v. 150, p. 729-746, 2013. https://doi.org/10.1016/j.jep.2013.09.026

Medeiros, P. M.; Ferreira Júnior, W. S.; Ramos, M. A.; Silva, T. C.; Ladio, A. H.; Albuquerque, U. P. Why do people use exotic plants in their local medical systems? A systematic review based on Brazilian local communities. PLoS ONE, v. 12, n. 9, e0185358, 2017. https://doi.org/10.1371/journal.pone.0185358

OMS - Organização Mundial de Saúde. Classificação estatística internacional de doenças e problemas relacionados à saúde. São Paulo: EDUSP, 2000.

Prado, D. E. As caatingas da América do Sul. In: Leal, I. R.; Tabarelli, M.; Silva, J. M. C. (Eds.). Ecologia e conservação da Caatinga. Recife: Editora Universitária da UFPE, 2003. p. 3-76.

Pedrosa, K. M.; Lopes, S. F.; Carvalho, T. K. N.; Lucena, C. M.; Nascimento, A. M. L.; Lucena, R. F. P. Traditional management of Cactaceae by local populations in the semi-arid region of Brazil. Ethnobiology and Conservation, v. 9, n. 28, p.1-13, 2020. https://doi.org/ 10.15451/ec2020-05-9.28-1-13

Quinlan, M. B.; Quinlan, R. J. Modernization and medicinal plant knowledge. Medical Anthropology, v. 21, n. 2, p. 169-192, 2007. https://doi.org/10.1525/maq.2007.21.2.169

Ribeiro, J. E. S.; Carvalho, T. K. N.; Ribeiro, J. P. O.; Guerra, N. M.; Silva, N.; Pedrosa, K. M.; Alves, C. A. B.; Sousa Júnior, S. P.; Souto, J. S.; Nunes, A. T.; Lima, J. R. F.; Oliveira, R. S.; Lucena, R. F. P. Ecological apparency hypothesis and availability of useful plants: Testing different use values. Ethnobotany Research \& Applications, v. 12, p. 415-432, 2014.

Sousa, F. S.; Cruz, K. C. M.; Souza, M. M. R. Congo. In: Lima, A. G.; Sobrinho, A. M.; Farias, C. M. P.; Freitas, D. K.; Batista, F. A.; Cruz, K. C. M.; Andrade, V. S. (Orgs.). Traços históricos e culturais do Cariri Paraibano. João Pessoa: s. ed., 2012. 
Tardío, J.; Pardo-de-Santayana, M. Cultural Importance Indices: A comparative analysis based on the useful wild plants of Southern Cantabria (Northern Spain). Economic Botany, v. 62, p. 24-39, 2008. https://doi.org/10.1007/s12231-007-9004-5

Trentin, D. D. S.; Giordani, R. B.; Zimmer, K. R.; Silva, A. G.; Silva, M. V.; Correia, M. T. S.; Baumvol, I. J. R.; Macedo, A. J. Potential of medicinal plants from the Brazilian Semi-Arid Region (Caatinga) against Staphylococcus epidermidis planktonic and biofilm lifestyles. Journal of Ethnopharmacology, v. 137, n. 1, p. 327-335, 2011. https://doi.org/10.1016/ j.jep.2011.05.030

Troter, R.; Logan, M. Informant consensus: A new approach for identifying potentially effective medicinal plants. In: Etkin, N. L. (Ed.). Indigenous medicine and diet: Biobehavioural approaches. New York: Redgrave Bedford Hills, 1986. p. 91-112.

Ullah, R.; Alqahtani, A. S.; Noman O. M. A.; Alqahtani, A. M.; Ibenmoussa, S.; Bourhia, M. A review on ethno-medicinal plants used in traditional medicine in the Kingdom of Saudi Arabia. Saudi Journal of Biological Sciences, v. 27, p. 2706-2718, 2020

Zappi, D.; Taylor, N. P.; Santos, M. R.; Larocca, J. Cactaceae. In: Lista de Espécies da Flora do Brasil. Rio de Janeiro: Jardim Botânico do Rio de Janeiro, 2018. Disponível em: <http://floradobrasil.jbrj.gov.br/jabot/floradobrasil/FB1750>. Acesso em: 25 out. 2020.

Zeni, A. L. B.; Bosio, F. Uso de plantas medicinais em uma comunidade rural de Mata Atlântica - Nova Rússia, SC. Netropical Biology and Conservation, v. 6, n. 1 p. 55-63, 2011. https://doi.org/10.4013/nbc.2011.61.07

Informação da Licença: Este é um artigo Open Access distribuído sob os termos da Licença Creative Commons Attribution, que permite uso irrestrito, distribuição e reprodução em qualquer meio, desde que a obra original seja devidamente citada. 